\title{
Rethinking complex diseases: A high-dimensional representation of individual disease burden and global disease landscape
}

Annikka Polster, MD PhD ${ }^{1}$

1) University of Oslo, Faculty of Medicine, Nordic Center for Molecular Medicine, Oslo 0318, Norway

\section{Visual Abstract}

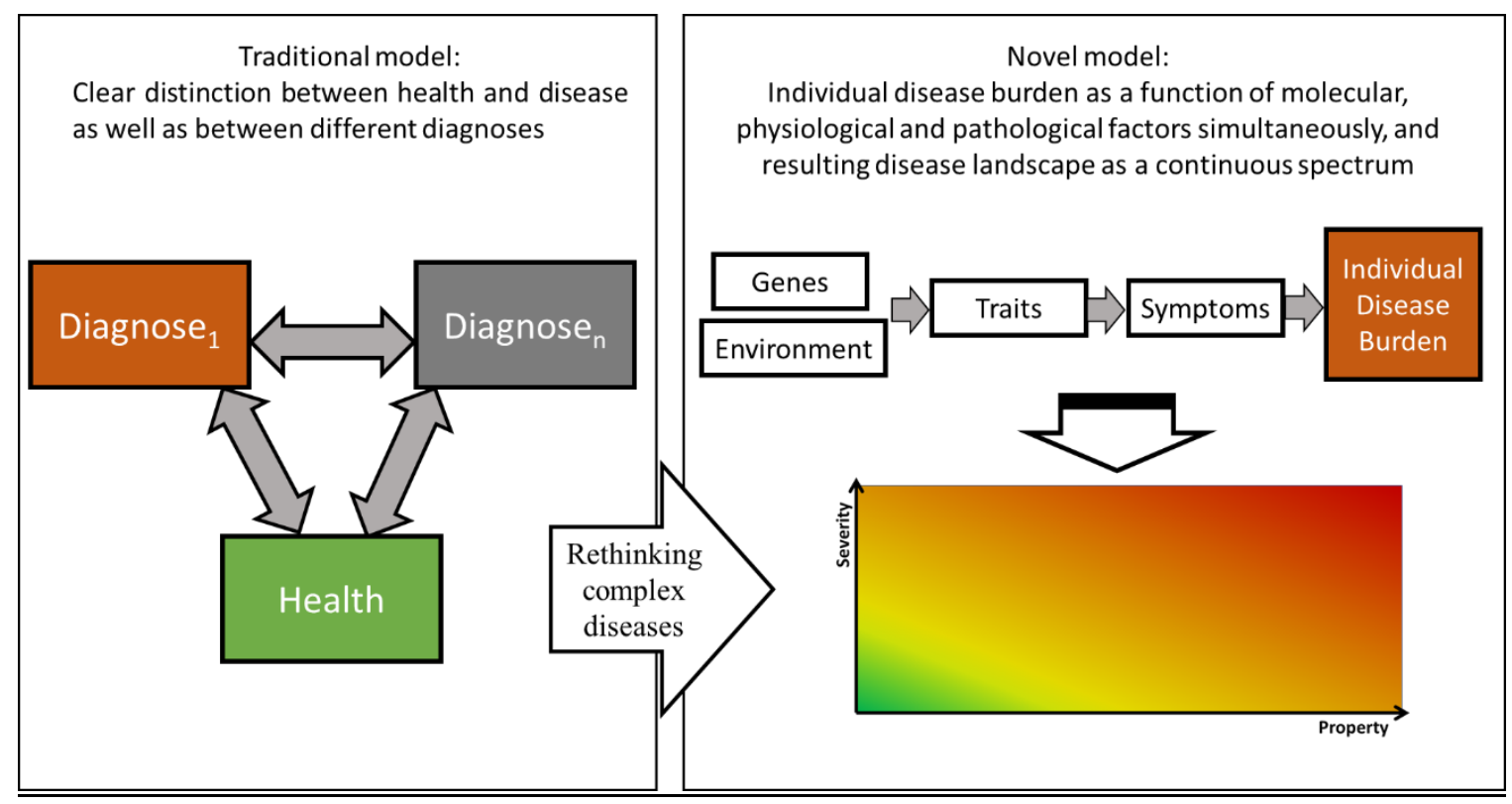

\section{$\underline{\text { Abstract }}$}

Complex diseases are prevalent medical conditions which are characterized by strong interpatient differences in symptom profiles, disease trajectory and treatment response. The challenges in understanding and managing these diseases are due to their complex pathophysiology, comprising of a combination of genetic and environmental factors. The traditional model of disease assumes a clear distinction between health and disease, as well as between different diagnoses, but recent findings with regards to diseasomes and network pleiotrophy suggest that this dogma is not useful in understanding complex diseases. This paper presents a novel model, in which the individual disease burden is determined as a function of molecular, physiological and pathological factors simultaneously: disease(symptoms(traits(genes AND environment))). From this a high-dimensional space is defined which includes all 
individual disease burdens, ranging from healthy (i.e. low disease burden) to multi-morbidity (i.e. high disease burden), termed the disease landscape. This model provides a novel way to conceptualize human physiology and pathophysiology in the context of complex diseases and may present a useful concept to simultaneously address the strong interindividual heterogeneity of diagnose cohorts as well as the lack of clear distinction between diagnoses and health and disease, thus facilitating the progression towards personalized medicine.

\section{Terminology}

Medical condition: Broad term that includes all diseases, injuries and syndromes.

Diagnosis: Classification of a disease based on the current body of knowledge in the medical field.

Complex disease: Medical condition with complex pathophysiology consisting of a combination of intrinsic and extrinsic factors.

Disease/disorder: A medical condition that is not due to any acute external injury. No unambiguous, generally accepted definition exists.

Ideally the term disease would describe a characteristic set of features/traits/symptoms with distinct identified pathophysiology and be synonymous to "diagnosis", but in the context of complex diseases this does not seem to be given.

Phenotype: The set of observable characteristics in an individual. Note: Is expanded when new measurement methods become available.

Clinical phenotype: The presentation of medical condition (one or several) in an individual.

Disease phenotype: Any observable characteristic or trait of a disease, such as symptoms, morphology, biochemical/physiological properties, behavior, without any implication of a mechanism. 


\section{Introduction}

Complex diseases, among which are highly frequent conditions such as neurodegenerative, metabolic and autoimmune diseases, are one of the major challenges of modern-day medicine. They are commonly incompletely understood and characterized by strong inter-patient differences in symptom profiles, disease trajectory and treatment response, leading to suboptimal disease management, reduced quality of life (e.g. (1)) and high healthcare costs (e.g. (2)). The challenges in understanding and managing these diseases are due to their complex pathophysiology, comprising of a combination of genetic and environmental factors.

Traditionally, complex diseases are viewed as distinct entities that are independent from each other, with the assumption that for each diagnosis distinct pathophysiological mechanisms, common to all patients with this diagnosis, can be identified and subsequently treated. In line with this assumption, patients suffering from several diagnosis are mostly conceptualized as having several simultaneously active distinct pathomechanisms, which need to be addressed separately. In recent years this view has become more differentiated in clinical practice, as it has become clear that many diagnoses of complex diseases actually represent umbrella terms for a cluster of similar clinical phenotypes containing subgroups of patients with differences in comorbidities, pathomechanisms, trajectories and the like (e.g. (3)). The understanding of these diagnoses and treatment options have therefore remained suboptimal. Attempts to molecularly characterize complex diseases and thus facilitate precise diagnostics and subtyping, for example through biomarker signatures or genome-wide association studies, have largely remained disappointing, as they lack reproducibility, predictive value or show missing heritability (4).

An interesting approach to thinking about diagnoses are diseasomes, which are attempts to link diagnoses via shared characteristics, for example via associated genetic factors (5) or shared symptoms (6). This way of thinking about disease partially addresses the lack of clear boundaries between different diagnoses and lack of specificity of potential pathomechanisms, as well as the importance of pleiotrophic effects on both clinical and disease phenotypes.

Another important concept to consider, especially with regards to missing heritability, is the poly-/omnigenic model (7), which reflects a growing body of evidence suggesting that complex 
traits are determined by a very large number of, or all genes expressed in the relevant tissue. Progressing this thought with regards to health and disease, it is easy to argue that most symptoms are determined by a combination of (at least) many traits. Pain perception, for example, can be considered to be determined by nociceptor characteristics, local and global immune regulators, afferent and efferent neural signaling and central processing, as well as sociocultural and psychological factors, and likely by many more (latent) traits. When further continuing this thought, it becomes clear that, for a large number of these traits, specific characteristics will be shared by people without similar symptom burden, or no symptoms at all. The same holds for the genes and alleles underlying the respective characteristics. The World Health Organization currently defines health as "a state of complete physical, mental and social well-being and not merely the absence of disease or infirmity" (8). Unfortunately, in practice this is not easy to apply, as the advance of diagnostic options (for example: screening) and the extent of their utilization, as well as changes in the criteria of abnormality defined by medical profession change the practical definitions of health and thus the proportion of healthy $v s$. diseased individuals in a population. This is important to consider, as it is not clear how to classify a) individuals with disease-alike abnormalities but who do not feel ill, b) individuals without measurable abnormalities but who do feel ill and c) individuals whose symptoms are managed and who are able to function adequately, but whose underlying pathologies remain unaltered.

Taken together, the unclear distinction between diagnoses as well as between health and disease pose a large hurdle in progressing our understanding of the mechanisms underlying complex disease onset, trajectory and treatment response. It is thus important to rethink the concepts underlying our current understanding of such medical conditions. This paper proposes a new way of thinking about complex diseases by considering individual disease burden and overall disease landscape, which may facilitate future research and targeted medical care by overcoming the limitations inherent to the current reductionist approaches.

\section{Disease landscape}


Grouping medical conditions into distinct diagnoses with matched treatment plans is currently clinically desirable, but the underlying assumptions remain dogmatic. It may thus be time to challenge this dogma and rethink complex diseases from a new viewpoint.

Instead of thinking about distinct diagnoses and searching for details discriminating disease from non-disease or diagnose A from diagnose B, it may be helpful to consider the individual disease burden as a function of symptoms, traits, genes and environmental factors simultaneously. This disease burden changes over time in response to external influences, such as lifestyle, mutations, ageing, therapeutic intervention, and the like. The respective individual disease burdens, in this conceptual model, form a disease landscape (Figure 1), in which individuals are located based on property (i.e. distinctive attributes or characteristics) and severity of the respective disease burden, and which ranges from no burden (equivalent to health) to diverse property and/or severity (the latter would be equivalent to severe disease and/or multi-morbidity).

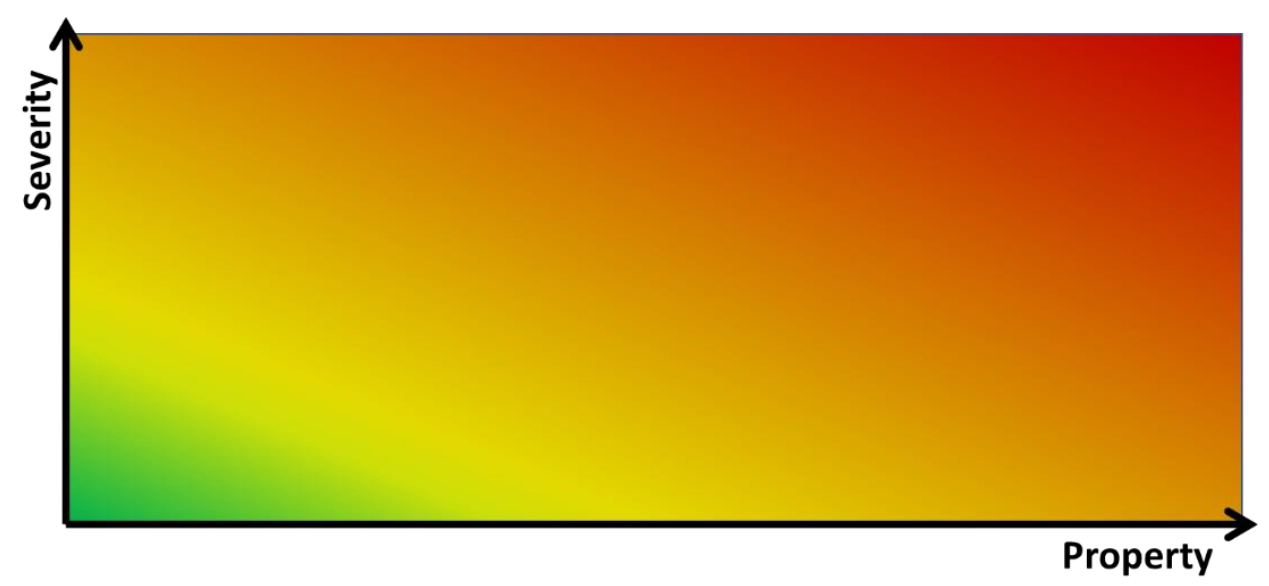

Figure 1: The disease landscape as a summary of property and severity, visualized as as a projection of the high-dimensional space including all individual disease burdens, ranging from healthy (i.e. low disease burden, green) to multi-morbidity (i.e. high disease burden, red).

\section{$\underline{\text { Individual disease burden }}$}

While diagnoses are defined through diagnostic criteria, disease itself is considered the absence of health defined as complete physical, mental and social well-being (8). Disease can thus be defined as a function of the symptoms presented by the respective person: 


\section{disease(symptoms)}

Symptoms in itself are in most cases, as outlined above, not single traits but rather the result of a combination of many to all traits of an individual, and are thus in this model defined as:

$$
\text { symptoms(traits). }
$$

Traits in turn are in this model understood as a function of genetic features in the context of environmental factors which alter transcription:

\section{traits(genes AND environment)}

Taken together, this results in the following formalization, where state vectors are utilized as descriptors of molecular and physiological characteristics, thus allowing for a high-dimensional representation of individual disease burden:

\section{disease(symptoms(traits(genes AND environment)))}

The result of this conceptual model is a novel view on human physiology and pathology, which will facilitate in-depth characterization of individuals outside of reductionist diagnostic confinements, and which facilitates viewing the overall disease landscape as a spectrum defined by individual disease property and severity.

\section{$\underline{\text { Discussion }}$}

This paper provides a novel way to conceptualize human physiology and pathophysiology in the context of complex diseases via individual disease burden and disease landscapes. This may be a useful concept to simultaneously address the strong interindividual heterogeneity of diagnose cohorts as well as the lack of clear distinction between diagnoses and health and disease. The intention is to present this concept and spark a discussion to establish novel ways to address the limitations of current nosological and epidemiological definitions. By refining the presented concept and using real-life data to determine individual disease burdens and evaluate the resulting disease landscape novel insights into human pathophysiology will be established and a progression towards personalized medicine facilitated. 


\section{Acknowledgements}

I would like to thank Prof. Lena Ohman and Prof. Marija Cvijovic for valuable input and discussions.

Competing interests: The author has no competing interests

\section{$\underline{\text { References }}$}

(1) Wilson A, Longstreth GF, Knight K, Wong J, Wade S, Chiou CF, Barghout V, Frech F, Ofman JJ. Quality of life in managed care patients with irritable bowel syndrome. Managed care interface. 2004 Feb 1;17(2):24-8.

(2) Park KT, Ehrlich OG, Allen JI, Meadows P, Szigethy EM, Henrichsen K, Kim SC, Lawton RC, Murphy SM, Regueiro M, Rubin DT. The cost of inflammatory bowel disease: an initiative from the Crohn's \& Colitis Foundation. Inflammatory bowel diseases. 2020 Jan 1;26(1):1-0.

(3) Polster A, Van Oudenhove L, Jones M, Öhman L, Törnblom H, Simren M. Mixture model analysis identifies irritable bowel syndrome subgroups characterised by specific profiles of gastrointestinal, extraintestinal somatic and psychological symptoms. Alimentary pharmacology $\&$ therapeutics. 2017 Sep;46(5):529-39.

(4) Manolio TA, Collins FS, Cox NJ, Goldstein DB, Hindorff LA, Hunter DJ, McCarthy MI, Ramos EM, Cardon LR, Chakravarti A, Cho JH. Finding the missing heritability of complex diseases. Nature. 2009 Oct;461(7265):747-53.

(5) Goh KI, Choi IG. Exploring the human diseasome: the human disease network. Briefings in functional genomics. 2012 Nov 1;11(6):533-42.

(6) Hoehndorf R, Schofield PN, Gkoutos GV. Analysis of the human diseasome using phenotype similarity between common, genetic and infectious diseases. Scientific reports. 2015 Jun $8 ; 5(1): 1-4$.

(7) Boyle EA, Li YI, Pritchard JK. An expanded view of complex traits: from polygenic to omnigenic. Cell. 2017 Jun 15;169(7):1177-86. 
(8) Constitution of WHO. Available from: https://www.who.int/about/governance/constitution. Accessed January 11, 2022 